\title{
Coronary atherosclerosis profile in patients with end-stage liver disease prior to liver transplantation due to alcoholic fatty liver: a coronary CTA study
}

\author{
Fabian Steinkohl ${ }^{1,2}$ (1) Fabian Barbieri $^{3} \cdot$ Thomas Senoner $^{3} \cdot$ Sylvia Strobl ${ }^{1} \cdot$ Armin Finkenstedt $^{4} \cdot$ Fabian Plank $^{3}$. \\ Christian Langer $^{1} \cdot$ Christoph Beyer $^{1} \cdot$ Katharina Birkl $^{1} \cdot$ Gerlig Widmann $^{1} \cdot$ Heinz Zoller $^{4} \cdot$ Wolfgang Dichtl $^{3}$. \\ Guy Friedrich $^{3} \cdot$ Herbert Tilg $^{4} \cdot$ Gudrun Feuchtner $^{1}$
}

Received: 13 November 2019 / Revised: 13 February 2020 / Accepted: 16 June 2020 / Published online: 4 August 2020

(C) The Author(s) 2020

\begin{abstract}
Objectives To assess the coronary atherosclerosis profile by coronary computed tomography angiography (CTA) in patients with end-stage liver disease (ESLD) due to alcohol-related liver disease (ARLD) evaluated for liver transplantation (LT), in a retrospective matched case-controlled cohort study.

Methods One hundred forty patients (age 60.6 years $\pm 9.8,20.7 \%$ females) who underwent coronary CTA were included. Seventy patients with ESLD due to ARLD (ESLD-alc) were propensity score (1:1) matched for age, gender, and the major 5 cardiovascular risk factors with healthy controls. CTA analysis included the following: stenosis severity according to CAD-RADS as $(0)=$ no, (1) minimal <25\%, (2) mild 25-50\%, (3) moderate 50-70\%, and (4) severe $>70 \%$ stenosis, total mixed plaque burden weighted for non-calcified component (G-score) and high-risk plaque criteria (Napkin-Ring, low attenuation plaque, spotty calcification, positive remodeling).

Results Prevalence of coronary artery disease (CAD) was high (84.4\%) in the ESLD-alc group but similar to controls. Stenosis severity was similar (CAD-RADS, 1.9 vs. $2.2, p=0.289)$. High-grade stenosis $(>70 \%)$ was observed in $12.5 \%$ of ESLD-alc patients. High-risk plaques were less frequent in the ESLD-alc cohort as compared to controls $(4.5 \%$ vs. $37.5 \%, p<0.001)$, and total mixed plaque burden was lower (G-score, 4.9 versus $7.4, p=0.001)$. Plaque density was lower in controls $(56.6 \mathrm{HU} \pm 3.2$ vs. $91.3 \mathrm{HU} \pm 4.5, p=0.007)$ indicating more lipid-rich in controls, but higher mixed fibro-calcific plaque component in those with alcohol-related ESLD.

Conclusion Patients with alcohol-related ESLD exhibit more mixed fibro-calcified plaques but less plaque with high-risk features and less fibro-fatty plaque burden, while total CAD prevalence is high.

Key Points

- Patients with ESLD prior to LT have a high total prevalence of CAD and stenosis severity, which is similar to those of healthy controls with an identical cardiovascular risk profile.

- Patients with ESLD prior to LT due to alcohol abuse have more calcific but less fibro-fatty plaque and less high-risk plaque.

- CTA seems to be a useful imaging technique for risk stratification prior to LT.
\end{abstract}

Keywords Angiography, computed tomography $\cdot$ Coronary artery disease $\cdot$ End-stage liver disease $\cdot$ Alcohol abuse $\cdot$ Risk factors

Fabian Steinkohl

Fabian.Steinkohl@i-med.ac.at

1 Dept. Radiology, Medical University of Innsbruck, Anichstr. 35, A-6020 Innsbruck, Austria

2 Radiology, St. Vincent Hospital Zams, Zams, Austria
3 Dept. Internal Medicine III, Cardiology, Medical University of Innsbruck, Innsbruck, Austria

4 Dept. Internal Medicine I, Gastroenterology, Hepatology and Endocrinology, Medical University of Innsbruck, Innsbruck, Austria 


$\begin{array}{ll}\text { Abbreviations } & \\ \text { ACS } & \text { Acute coronary syndrome } \\ \text { AHA } & \text { American Heart Association } \\ \text { ARLD } & \text { Alcohol-related liver disease } \\ \text { AU } & \text { Agatston units } \\ \text { BMI } & \text { Body mass index } \\ \text { CABG } & \text { Coronary artery bypass graft } \\ \text { CAD } & \text { Coronary artery disease } \\ \text { CAD-RADS } & \text { Coronary Artery Disease-Reporting and Data } \\ & \text { System } \\ \text { CCS } & \text { Coronary calcium score } \\ \text { cMPR } & \text { Curved multiplanar reformations } \\ \text { CRP } & \text { C-reactive protein } \\ \text { CTA } & \text { Computed tomography angiography } \\ \text { CVD } & \text { Cardiovascular disease } \\ \text { EF } & \text { Ejection fraction } \\ \text { ESLD } & \text { End-stage liver disease } \\ \text { GFR } & \text { Glomerular filtration rate } \\ \text { HR } & \text { Hazard ratio } \\ \text { HU } & \text { Hounsfield units } \\ \text { INR } & \text { International normalized ratio } \\ \text { LAP } & \text { Low attenuating plaque } \\ \text { LT } & \text { Liver transplantation } \\ \text { LVEF } & \text { Left ventricular ejection fraction } \\ \text { MACE } & \text { Major adverse cardiac event } \\ \text { MELD } & \text { Model for end-stage liver disease } \\ \text { NRS } & \text { Napkin-ring sign } \\ \text { NCP } & \text { Non-calcified plaque } \\ \text { RI } & \text { Remodeling index } \\ \text { ROI } & \text { Region of interest } \\ \text { SD } & \text { Standard deviation } \\ & \end{array}$

\section{Introduction}

Cardiac complications are a frequent cause of death after liver transplantation (LT) [1]. Therefore, preoperative cardiac risk stratification is needed in patients, who are listed for LT. It seems that coronary calcium score (CCS) [2] and coronary computed tomographic angiography (CTA) are safe and useful tools for this task [3].

It is known that moderate amounts of alcohol have protective effects on coronary artery disease (CAD) [4]. Heavy drinking may adversely affect the cardiovascular system, but there is controversy in the literature [5, 6]. Patients with endstage liver disease (ESLD) due to excessive alcohol consumption form a large cohort of all LT patients. The direct effects of excessive alcohol consumption on the coronary atherosclerosis profile in these patients prior to liver transplantation have not yet been investigated in a study using coronary computed tomography angiography (CTA).

Coronary CTA is a highly accurate quantification tool for coronary atherosclerosis in terms of stenosis severity (CAD-
RADS) [7] and total (including non-calcified and mixed) plaque burden, and enables the characterization of "high-risk" vulnerable plaque features, such as the napkin-ring sign (NRS), low attenuation fibro-fatty plaque (LAP), spotty calcification, and positive remodeling. These "high-risk" plaque markers have recently emerged as indicators for increased risk of major cardiovascular events (MACEs) [8-10].

Therefore, the purpose of our study was to investigate coronary atherosclerosis characteristics in patients with ESLD due to alcohol-related liver disease (ARLD) and a documented history of alcohol abuse (ESLD-alc) [11], in a retrospective matched case control cohort study.

\section{Methods}

\section{Study population}

All patients with ESDL referred for CAD screening prior to LT were extracted from our coronary CTA database consisting of patients referred to coronary CTA between 2006 and 2016. Then, patients with a history of an alcohol use disorder [12] documented in hospital information system were included into the "ESLD-alc" group. Those were propensity score (1:1) matched with non-regular alcohol drinking individuals without known liver disease.

Conventional coronary risk factors according to standardized European Society of Cardiology (ESC) criteria were collected: arterial hypertension (systolic blood pressure $>140 \mathrm{mmHg}$ or diastolic blood pressure $>90 \mathrm{mmHg}$ ), dyslipidemia (total cholesterol $>200 \mathrm{mg} / \mathrm{dl}$ or $\mathrm{HDL}<40 \mathrm{mg} / \mathrm{dl}$ ), family history (myocardial infarct or sudden cardiac death in an immediate male relative $<55$ years or female $<65$ years), smoker (current or quit within the last 6 months), and diabetes.

The new model for end-stage liver disease (MELD) score was calculated as follows: $9.57 \times \ln ($ creatinine $\mathrm{mg} / \mathrm{dl})+$ $3.78 \times \ln ($ total bilirubin $\mathrm{mg} / \mathrm{dl})+11.2 \times \ln (\mathrm{INR})+6.43$, [11]. The Child-Pugh Stadium was recorded and classified into classes $\mathrm{A}, \mathrm{B}$, and $\mathrm{C}$.

\section{Inclusion criteria}

\section{ESLD-alc group}

- Evidence of alcohol abuse reported during psychiatric LT evaluation and/or liver histopathology (Mallory Weiss bodies).

- Meeting ESLD criteria [11] and fulfilling criteria for LT listing (MELD $\geq 15$ or clinical signs of decompensated liver cirrhosis like refractory ascites, hepatic encephalopathy, sarcopenia). Diagnosis of ARLD was made according to the current European guidelines [13].

- All patients were evaluated prior to LT. 


\section{Control group}

- Patients denied alcohol consumption by a questionnaire prior to CTA exam, or reported non-regular occasional drinking of maximum 1 glass of an alcoholic beverage maximal once per week.

- Normal liver enzymes.

\section{Exclusion criteria for coronary CTA}

- Renal dysfunction (GFR $<30 \mathrm{ml} / \mathrm{min}$ )

- Previous coronary artery bypass grafting (CABG) grafting

- Current acute coronary syndrome (ACS) or unstable angina

- Severe aortic stenosis

\section{Coronary computed tomography}

Coronary computed tomography angiography Non-contrast ECG-gated CCS with standardized scan parameters (detector collimation $64 \times 1.5 \mathrm{~mm} ; 120 \mathrm{kV}$ ) was performed. The Agatston Score was calculated [14]. Then, coronary CTA was performed with a retrospectively ECG-gated 64-slice CTA (GE Healthcare or Siemens Somatom Sensation 64) from 2005 to 2009, and from 2010 onwards with a 128-slice dual source CTA (Definition FLASH, Siemens) with a detector collimation of $2 \times 64 \times 0.6 \mathrm{~mm}$, a $z$-flying spot of $64 \times$ $0.6 \mathrm{~mm}$, and a rotation time of $0.28 \mathrm{~s}$. Prospective ECG triggering was used in regular heart rates $<65 \mathrm{bpm}$ (diastolic padding, $70 \%$ of RR interval); in heart rates $>65 \mathrm{bpm}$ and irregular heart rhythm, retrospective ECG-gating was applied.

An iodine contrast agent (iopromide, Ultravist 370, Bayer Pharma $A G$ ) was injected intravenously (flow rate $4-6 \mathrm{ml} / \mathrm{s}$ followed by $40-\mathrm{ml}$ saline chaser), triggered into arterial phase (bolus tracking; $100 \mathrm{HU}$ threshold; ascending aorta). Contrast volume ranged from 65 to $120 \mathrm{ml}$, and was adjusted to the individual patient characteristics using a standardized scheme. Axial images were reconstructed with $0.75-\mathrm{mm}$ slice width (increment 0.4/medium-smooth kernel B26f) during best diastolic and systolic phase. The same CT protocol was used for both groups.

CTA image analysis Curved multiplanar reformations (cMPRs) and oblique interactive MPR of all vessels using 3D post-processing software (SyngoVia ${ }^{\mathrm{TM}}$, Siemens Healthineers) were generated:

1) Coronary stenosis severity was scored as follows: minimal $<25 \%$, mild $\leq 25-49 \%$, intermediate $50-69 \%$, or severe $\geq 70 \%$ according to CAD-RADS [15] per coronary segment (American Heart Association (AHA)-modified16-segment classification) [16].

2) The coronary plaque types were characterized as: calcified (T1), mixed (dominantly calcified $>$ non-calcified) (T2), mixed (dominantly non-calcified $>$ calcified) (T3), non-calcified (T4) per coronary segment. Calcified and non-calcified plaque components were defined as hyperand hypoattenuating lesions with $>$ and $<150 \mathrm{HU}$ [10]. The G-score [17] (= sum of plaque types T1-T4 for each segment), as radiologic marker for an increased noncalcifying plaque burden, per coronary segment (AHAmodified-16-segment classification) was calculated.

3) Quantitative High-risk plaque (HRP) analysis [8]

- Non-calcified plaque (NCP) was defined as being hypodense as compared to vessel lumen, < $150 \mathrm{HU}$ [18]. CT density was screened by utilizing "pixel-lens" [16] and the minimal HU was recorded. Then, an area ROI was placed into the lowest density plaque area, and drawn as large as possible, while sparing areas affected by artifacts.

- Low attenuation plaque $(L A P)<60 \mathrm{HU}$ was defined as fibro-fatty plaque and regarded as HRP criterion [10].

- Napkin-ring sign (NRS) was defined as hyperdense rim surrounding a hypodense plaque core [9].

- Spotty calcification was defined as calcification $<3-\mathrm{mm}$ size within a NCP.

- The remodeling index $(R I)$ was calculated as the ratio of the maximal cross-sectional vessel diameter including the plaque and the lumen, and its closest proximal (or in ostial lesions distal) normal reference vessel lumen diameter. An RI was defined as positive if $>1.1$.

If a patient had multiple lesions, all lesions were quantified separately and counted.

A high-risk plaque was defined as such: if a minimum of 2 of 4 criteria were present [10].

Coronary CTA image analysis was performed by one observer ( $>10$ years' experience) and a second independent observer with $>6$ months of training. Consensus reading was obtained.

\section{Statistical analysis}

Statistical analysis was performed using IBM SSPSTM software (V 25.0, IBM Corporation). Propensity score matching was performed by 1:1 method for age, gender, body mass index (BMI), and the major 5 risk factors (arterial hypertension, smoking, positive family history, dyslipidemia, and diabetes).

Quantitative variables were expressed as mean \pm standard deviation (SD); categorical variables reported as absolute values and percentages. Normal distribution was tested with Kolmogorov-Smirnov test. 
Mean differences between parametric data were tested by using the appropriate tests according to their distribution $(t$ test for normally, and Mann-Whitney $U$ for non-normally distributed and rank-scale data such as G-score, segment involvement score, CAD-RADS, and CCS). Differences in categorical data (gender, coronary risk factors, prevalence of CAD, CAD-RADS 0-4, high-risk plaque) between groups were determined with chi-square or Fisher's exact test (if $n \leq 5$ per group). A $p$ value of less than 0.05 was considered significant.

\section{Results}

A total of 488 patients with ESLD listed for LT were extracted from our coronary CTA database consisting of 9988 patients. Then, those patients with a history of alcohol abuse were selected $(n=97)$ and propensity score matched 1:1 with patients from our database consisting of 4400 patients, who denied regular alcohol consumption. Seventy-two patients with ESLD due to alcohol abuse could be matched and were enrolled. Two patients from each group were excluded; due to either inadequate CT image quality $(n=2)$ (high image noise or motion artifacts), or other exclusion criteria (severe aortic stenosis $(n=1)$ or previous CABG grafting $(n=1))$.

In total, 140 patients (70 with ESLD-alc and 70 controls) were included. Sixty-four underwent both coronary CTA and CCS. In 6 patients, only CCS was performed due to renal dysfunction.

Demographic and clinical characteristic are shown in Table 1. There was no significant difference with regard to age, gender, and the major five cardiovascular risk factors. The prevalence of previous acute renal injury was higher in ESLD as compared to controls. Left ventricular ejection fraction (LVEF) was normal in both groups; none of the subjects had severe heart failure with a LVEF of less than $35 \%$.

Table 2 shows coronary CTA results. Prevalence of CAD, defined as presence of plaque, was high but comparable between the ESLD-alc group and controls (84.4\% vs. $87.5 \%)$, as well as stenosis severity (CAD-RADS), which was similar between the two groups (1.9 vs. $2.2, p=0.289$ ). The prevalence of high-grade $>70 \%$ stenosis was $12.5 \%$ in ESLD-alc patients and did not differ significantly from controls.

Figure 1 shows the coronary CTA of a former drinker with ESLD and large vessel size even at side branch and septal branch level, indicative of vasodilatory effects, and minimal plaque burden (high-density fibrous plaque). Figure 2 presents a patient with high calcium score, who quitted drinking 2 years ago.

Total mixed plaque burden was lower in patients with ESLD due to prior alcohol abuse (G-score: mean 4.9 vs. 7.4, $p=0.001$ ). Figure 1 shows a patient with ESLD, low plaque burden, and a CCS of zero. Figure 2 illustrates a case of ESLD with high exclusively, calcified plaque burden.
Table 1 Study cohort

\begin{tabular}{lccr}
\hline & ESLD-alc & \multicolumn{2}{l}{ CR } \\
& $N=64$ & \multicolumn{1}{l}{$N=64$} & \\
& $60.8 \pm 9$ & $60.5 \pm 10$ & 0.850 \\
Age (years) & $8(12.5 \%)$ & $9(14.1 \%)$ & $>0.999$ \\
Females & $26.3( \pm 37)$ & $26.4( \pm 43)$ & 0.609 \\
BMI kg/m ${ }^{2}$ & $57(89 \%)$ & $60(93.7 \%)$ & 0.528 \\
Arterial HT & $36(56.2 \%)$ & $34(53.1 \%)$ & 0.859 \\
Smoking & $2(3.1 \%)$ & $3(4.6 \%)$ & $>0.999$ \\
Positive FH & $7(10.9 \%)$ & $8(12.5 \%)$ & $>0.999$ \\
Dyslipidemia & $9(14.0 \%)$ & $8(12.5 \%)$ & $>0.999$ \\
Diabetes & $15.7 \pm 6.0$ & & \\
MELD score & & & \\
Child-Pugh stage & $9(14.1 \%)$ & & \\
A & $34(53.1 \%)$ & & \\
B & $21(32.8 \%)$ & & \\
C & $12(18.7 \%)$ & $0(0 \%)$ & \\
Previous acute renal injury & $59.9 \pm 6.1$ & $63.6 \pm 12.8$ & \\
LV EF \% & $0(0 \%)$ & $0(0 \%)$ & \\
EF <35\% & & & \\
\hline
\end{tabular}

Abbreviations: $E F=$ ejection fraction. $L V=$ left ventricle. $M E L D=$ model for end-stage liver disease. $H A T=$ hypertension. $F H=$ family history. $B M I=$ body mass index. $n . s .=$ non-significant

High-risk plaques were markedly less common in the ESLD-alc group as compared to controls $(4.5 \%$ vs. $37.5 \%$, $p<0.001)$. Also the total number of high-risk plaques was lower (Fig. 3).

Plaque density was lower in controls as compared to the ESLD-alc group (56.6 $\mathrm{HU} \pm 3.2$ vs. $91.3 \pm 4.5)$, indicting more dense mixed fibro-calcific plaque burden in patients with alcoholic ESLD.

CCS was higher in ESLD patients (132.8 vs. $72.9 \mathrm{AU}, p=$ 0.673 ) but the difference did not reach statistical significance. The prevalence of non-calcified plaques in patients with a CCS of zero was similar between the two groups but reached $30.7 \%$ in ESLD.

\section{Discussion}

Our study shows lower non-calcified fibroatheroma and less high-risk plaque burden, but a trend to higher denser mixed calcified coronary plaque in patients with alcohol-related endstage liver disease (ESLD-alc), as compared to a matched cohort without liver disease and without alcohol consumption but an identical CV risk profile.

However, total prevalence of CAD was high and similar between both groups. The reasons for our findings could be explained as follows. 
Table 2 Coronary atherosclerosis profile by coronary CTA: 140 patients $(n=128$ with CTA and CCS, $n=12$ with coronary calcium score (CCS) only)

\begin{tabular}{lclc}
\hline & $\begin{array}{l}\text { ESLD-alc } \\
N=64\end{array}$ & $\begin{array}{l}\text { controls } \\
N=64\end{array}$ & $p$ value \\
\hline CAD $n(\%)$ & $54(84.4 \%)$ & $56(87.5 \%)^{*}$ & $0.465^{*}$ \\
CAD-RADS 1-2 & 32 & 35 & \\
CAD-RADS 3 & 13 & 10 & \\
CAD-RADS 4 & $8(12.5 \%)$ & $12(18.7 \%)$ & $0.289^{* *}$ \\
CAD-RADS & $2(\mathrm{IQR} 1)$ & $2(\mathrm{IQR} 2)$ & $0.001^{* *}$ \\
G-score & $8(\mathrm{IQR} 7)$ & $4(\mathrm{IQR} 7)$ & $<0.001^{*}$ \\
HRP $n(\%) *$ & $5(4.5 \%)$ & $24(37.5 \%)$ & $<0.001^{*}$ \\
\#HRP total $(n)$ & 6 & $56.6 \pm 3.2$ & $0.007^{* * *}$ \\
LAP density (HU) & $91.3 \pm 4.5$ & $95(\mathrm{IQR} 403)$ & $0.673^{* *}$ \\
Coronary Calcium Score (AU) & $107(\mathrm{IQR} 1046)$ & $10 / 17(58.8 \%)$ & $0.551^{*}$ \\
$N=70$ & $4 / 13(30.7 \%)$ & & \\
NCP in Calcium Score zero & & & \\
\hline
\end{tabular}

Abbreviations: $L A P=$ low attenuation plaque. $N C P=$ non-calcified plaque. $A U=$ Agatston Units. $N=$ counts. $H U=$ Hounsfield Units. $E S L D$-alc $=$ end-stage liver disease due to alcohol consumption

*per patient. \#HRP = number of HRP. *Chi-square; **Mann-Whitney $U$ (non-parametric); ***independent $t$ test Counts are displayed as numbers (N). For CADRADS, G-score, and CCS, the median and IQR (interquartile range) are shown
None of the patients with ESLD was actively and excessively drinking alcohol at the time of coronary CTA, since they were evaluated for LT and had to be abstinent for a minimum of 6 months, in order to be listed for surgery. This may partially explain the lack of high-risk plaque as an indicator for plaque inflammation. Laboratory pro-inflammatory parameters, which are usually increased during episodes of long-term active excessive drinking, will most likely downregulate after cessation of active drinking. The distinct atherosclerosis profile (with more calcific plaque) may be also relate to other pathways driving coronary calcification: Transient or chronic kidney dysfunction is common in ESLD, which enhances coronary calcification. Repeated episodes of arterial hypertension during heavy drinking as well most likely stimulate calcification progression.

While data on CAD burden in ESLD patients are sparse, there are some studies in patients with liver disease indicating the following trends.

Both steatosis and cirrhosis of the liver have been linked to a higher $\mathrm{CAD}$ burden, especially to a higher calcified plaque burden [19, 20]: In 2028 patients with steatosis, a liver attenuation of $<54 \mathrm{HU}$ was associated with obstructive CAD, independently of conventional cardiac risk factors. The severity of coronary calcification was associated with the severity of fatty liver [19].

In a smaller cohort of only 52 patients [20] with cirrhosis of all Child-Pugh stadiums, a high CAD prevalence (77\%) was found, which is comparable to the results in our cohort. CCS and severity of CAD in terms of vessel involvement as well as the volumes of non-calcified and calcified plaques were higher [20]. In contrast to our work, this study [20] consisted of a small heterogeneous cohort of patients with cirrhosis of varying etiology.

However, until now, no study has analyzed the coronary artery disease profile a specific cohort of patients with severe ESLD due to ARLD.

As novelty, we analyzed high-risk plaque criteria [7-10], while previous studies enrolled patients with ESLD of varying etiologies, and mainly calculated only the CCS [21]. CCS is a less sensitive and less accurate method for defining the coronary atherosclerosis profile as opposed to coronary CTA: Coronary CTA allows for stratification of coronary stenosis severity (CAD-RADS), total mixed plaque burden, and plaque morphology characterization, including the detection of "high-risk" plaque criteria [18].

The effect of alcohol consumption on heart diseases is controversially discussed in the literature. While the protective effects of moderate amounts of alcohol on coronary heart disease outcomes have been well studied in epidemiological trials [4], heavy drinking has been associated with increased risk of heart failure [22] due to higher left ventricular remodeling and mass [23].

In a recent meta-analysis, even higher amounts of alcohol ( $>7$ drinks/day) improved outcomes in patients with heart failure [5]. However, in another meta-analysis (13 prospective studies, 355,804 participants) [24], light alcohol drinking $(<7$ drinks/week) was inversely associated with risk of heart failure, while no statistically significant association between moderate (7-14 drinks/week), high (>14 drinks/week), or heavy ( $>28$ drinks/week) alcohol consumption and heart 
Fig. 1 A 64-year-old male with one risk factor (arterial hypertension) and ESLD due to alcohol-related liver disease. CTA shows a small non-calcified plaque in the proximal LAD (arrow) with a high plaque density (HU 80-90) and minimal stenosis (CAD-RADS 1). Calcium score was zero. VRT (left) and cMRP (right) of the RCA; LAD and CX
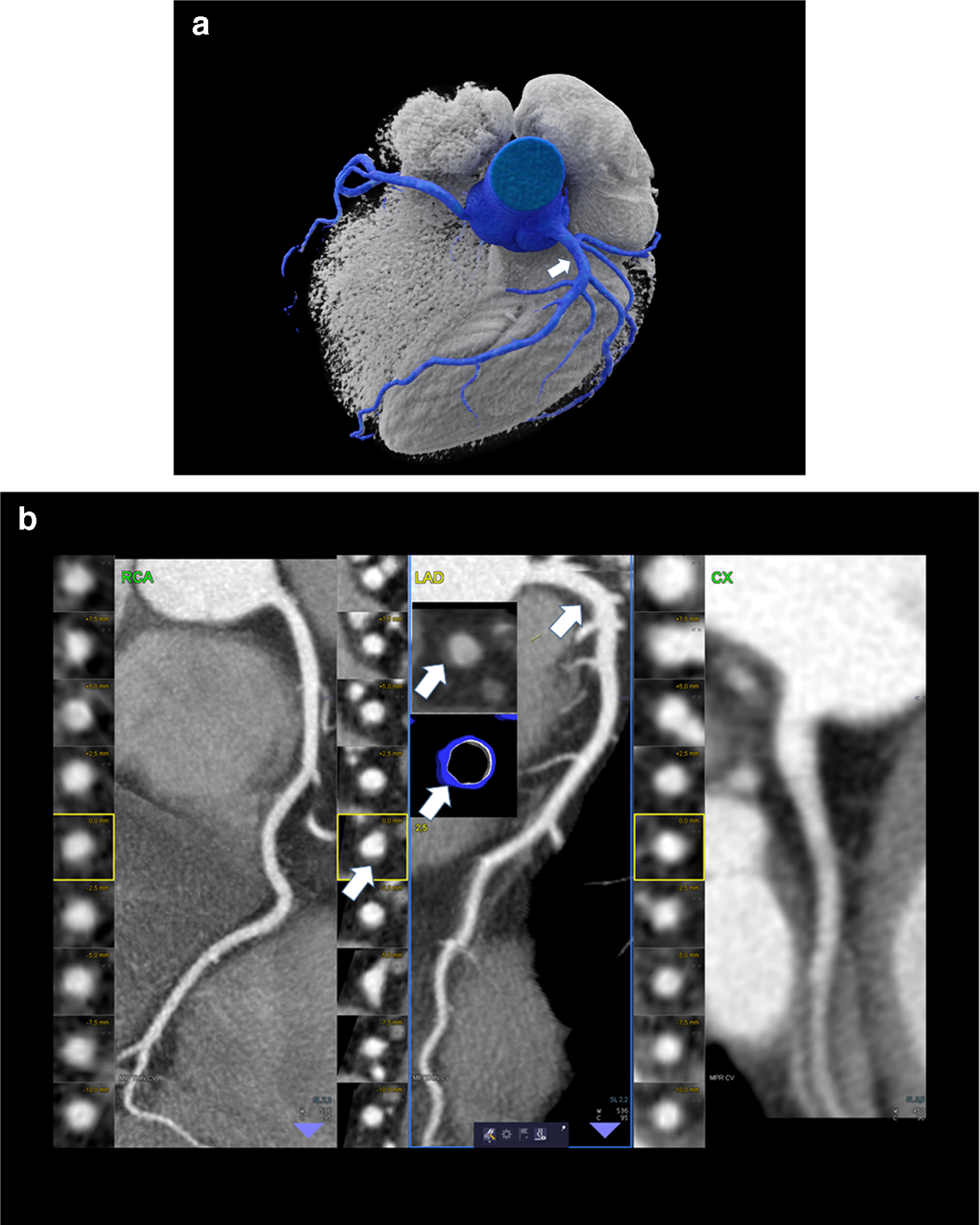

failure was found. Former drinking was associated with an increased risk of heart failure compared with never or occasional drinking [24].

In a recent meta-analysis [6] including 599,912 current drinkers, alcohol consumption was roughly linearly associated with a higher risk of stroke (hazard ratio (HR) per $100 \mathrm{~g}$ per week higher consumption 1.14), coronary disease excluding myocardial infarction (HR 1.06), and heart failure (HR 1.09) [6].

By contrast, increased alcohol consumption was loglinearly associated with a lower risk of myocardial infarction (HR 0.94). In comparison to those who reported drinking $>0-\leq 100 \mathrm{~g}$ alcohol per week, patients who drank more $(>100-\leq 200$ g per week, $>200-\leq 350$ g per week, or $>$
$350 \mathrm{~g}$ per week) had lower life expectancy at age 40 of approximately 6 months (and 1-2 years, or 4-5 years, respectively).

Studies investigating the effect of alcohol on cardiovascular outcomes have shown varying results, pending on which cohorts were enrolled (patients with heart failure or healthy patients) and which endpoints were used. On a physiological level, there may be beneficial effects from antioxidants (e.g., resveratrol from red wine) and from vasodilatative effects. Hence, different types of alcohol may be more or less beneficial on the cardiovascular system. However, high levels of alcohol consumption increase blood pressure and high endothelial sheer act pro-atherogenic and stimulate the calcification process. Further, toxic interactions could also act proatherogenic by inducing pro-inflammatory pathways. 
Fig. 2 A 57-year-old-male with 1 risk factor (arterial hypertension) and ESLD due to alcohol-related liver disease. CTA was performed 2 years after quitting alcohol abuse prior to liver transplantation. MELD score was 12, and Child-Pugh Stadium B and several episodes of liver decompensations were recorded. Cardiac Calcium Score was high with 527 AU. CTA showed multiple calcified plaques in the $\mathrm{RCA}$; LAD and $\mathrm{CX}$ with less than $50 \%$ stenosis (CAD-RADS 2). VRT (left) and cMRP (right) of $\mathrm{RCA}$; LAD and CX with multiple calcified but no high-risk plaque

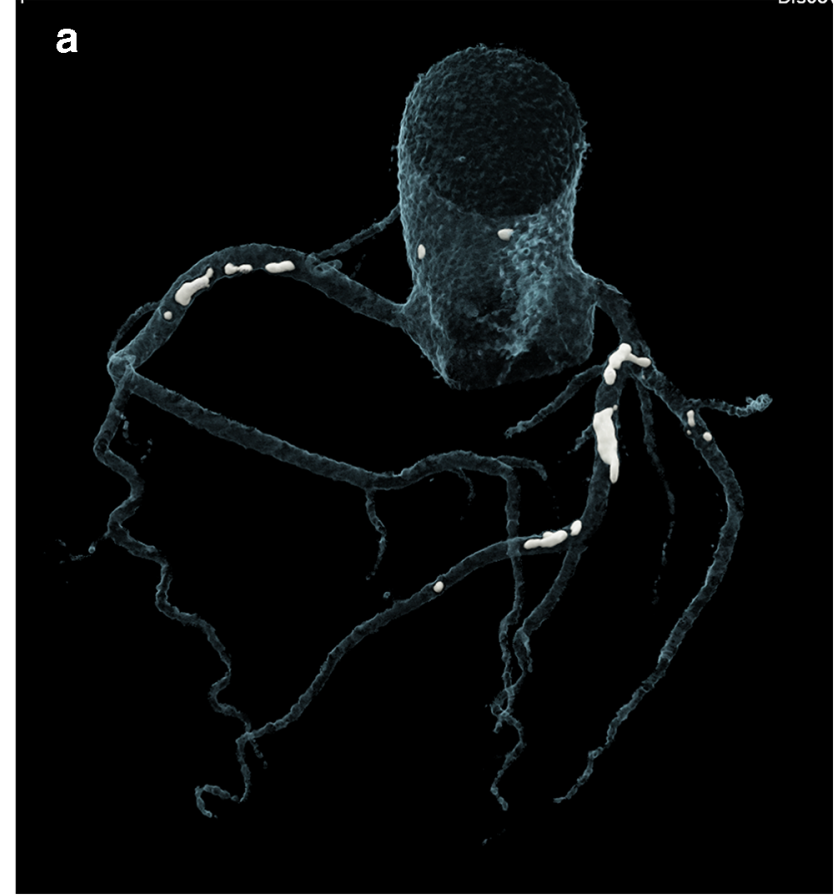

b

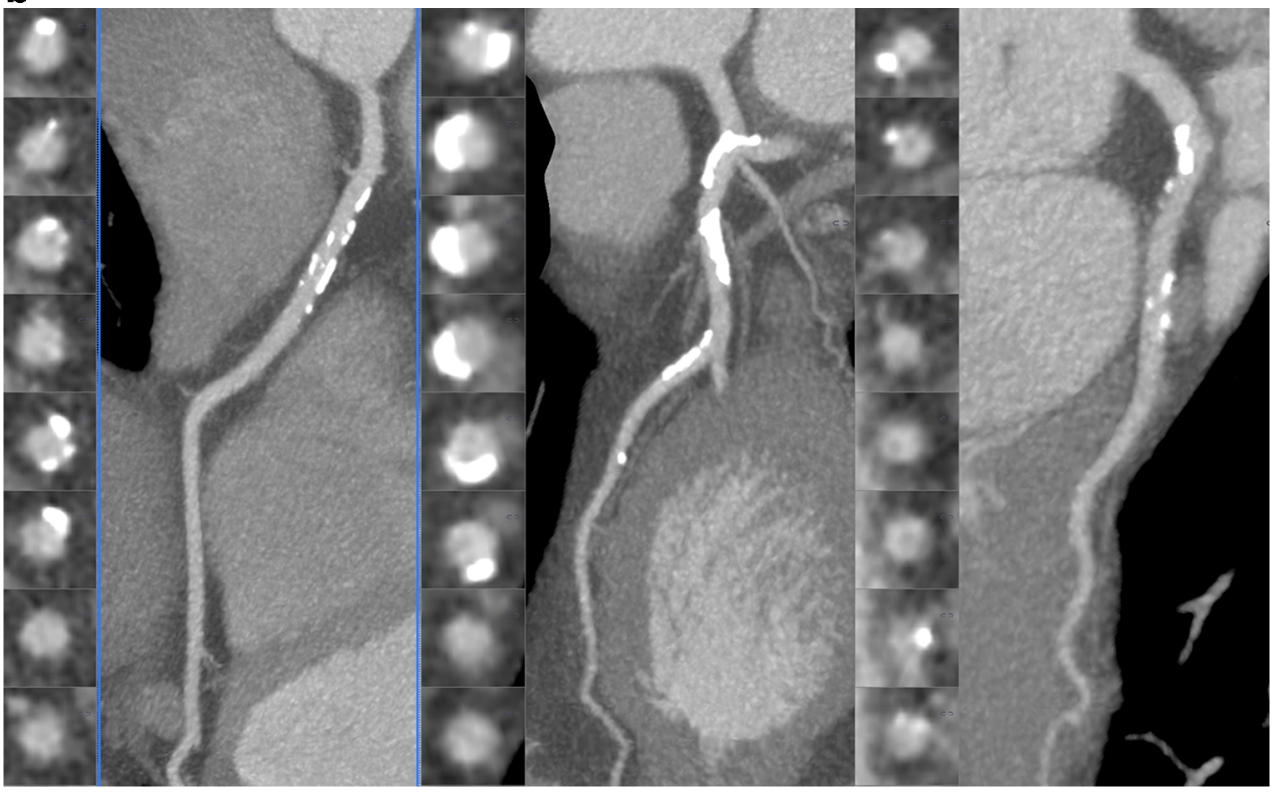

There is scientific evidence from epidemiologic studies (Women's Health Study, 26,000 participants) proving that alcohol consumption is an independent predictor of cardiovascular events [4], with a J-shape (dose-dependent) association between alcohol consumption and incident cardiovascular disease (CVD) and CVD mortality. Cardioprotective effects are supposed to be modulated with anti-inflammatory mechanisms although those mechanisms have never been fully understood [4].

However, inflammatory and hemostatic factors such as Creactive protein (CRP), soluble intracellular adhesion molecule-1, and fibrinogen were $21 \%$ lower in moderate alcohol drinkers (5-14.9 g/day) and 13\% lower in heavy drinkers (>15 g/day) compared to abstainers or occasional consumers of alcohol. Bektas et al. confirmed the absence of "inflammatory" stages of atherosclerosis [4]. This is in line with our findings, with regard to the low incidence of highrisk plaque features.

As a result, non-calcified lipid-rich plaque was found less often in drinkers as compared to "healthy" controls. Nonetheless, and surprisingly, about one third (30.7\%) of patients with CCS zero had non-calcifying plaque by CTA. This 
Fig. 3 A 47-year-old-male, 1 risk factor (arterial hypertension) who denied ever drinking alcohol (control group). Low coronary calcium score (4.1 Agatston Units) but high non-calcified plaque burden (LM and LAD, white arrow) with a high-risk plaque in the proximal LAD with low attenuation (44 HU) and napkin-ring sign (inlay, outer hyperdense rim)
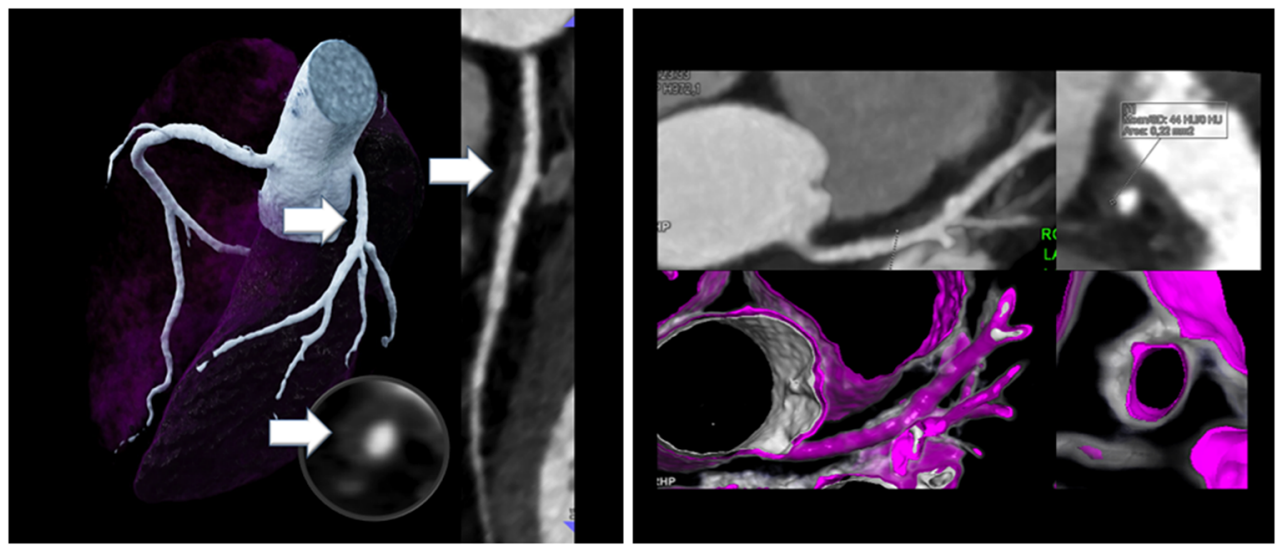

finding does not support using CCS for risk stratification prior to LT.

On one hand, other comorbidities related to advanced stages of liver disease may contribute to increased coronary calcium, such as renal dysfunction. On the other hand, increased estrogen levels in patients with ESLD may have rather protective effects on atherogenesis.

We did not find a higher prevalence of signs of plaque inflammation (high-risk plaque criteria) in our populations. This may be explained by the fact that our patients quit drinking before coronary CTA as they were evaluated for LT. Thus, there were no "active" heavy drinkers at the time of coronary CTA, in contrast to the meta-analysis on "active" high alcohol consumption [6]. High-risk plaques detected by coronary CTA are predictors of adverse outcome in terms of both MACE [25] and vessel-specific ischemia [26, 27].

Interestingly, a novel study published this month showed that along with increasing plaque density (HU), the risk of MACE declines. Especially the 1-K Plaque ( $>$ above 1000 $\mathrm{HU}$ ) had the lowest risk of MACE in the ICONIC registry and may improve risk stratification [28].

\section{Study limitations}

We acknowledge the retrospective study design with its inherent bias. Confounding risk factors were minimized by best medical practice (1:1 propensity score matching).

All patients had a history of previous excessive alcohol consumption, resulting in ESLD. Because they were evaluated for LT, they had to prove to be abstinent by regular controls of their urine ethyl glucuronide levels for at least 6 months and regular psychological evaluation.

The time between alcohol renouncement and CTA varied, since the time interval between quitting and coronary CTA ranged from a minimum of 6 months up to several years ( $>6$ years).

Of note, we would like to emphasize, that the CAD disease profile in our cohort is specific for ESLD patients prior to LT due to alcohol abuse, but may be distinct in patients with alcohol abuse without ESLD.

We acknowledge that a sub-analysis of the type of alcohol consumption cannot be provided due to the mixed drinking behavior and incomplete data collection.

Hard study endpoints (MACE; mortality) were not collected. Therefore, a conclusion, whether calcium scoring or coronary CTA is better for risk stratification prior to LT, is not possible. However, the high total prevalence of CAD in the ESDL cohort rather suggests the use of CTA, in order to identify individuals with significant stenosis, who require invasive coronary angiography and coronary intervention in order to avoid adverse outcomes after LT and intraoperative cardiovascular complications during LT. Coronary CTA has shown to be superior to CCS for CV risk stratification in numerous data outputs from CONFIRM registry [29, 30].

\section{Conclusion}

Patients with alcohol-related ESLD exhibit have a high total prevalence of CAD with a higher calcified plaque burden but less high-risk "vulnerable" plaque characteristics and a less lipid-rich plaque burden. Patients with ESLD have poor cardiovascular outcomes [31].

Our study sheds light on the mechanism of alcohol-related ESLD on the coronary arteries, in terms of reducing "highrisk" inflammatory plaque but increasing coronary calcification, while maintaining an overall high CAD burden.

In summary, our data support using CTA rather than calcium scoring for the evaluation of patients with ESLD prior to LT. The advantages of CTA comprise: Detection of CAD at early stages and adverse plaque characteristic [32], and diagnosis of significant stenosis [29, 30], and to initiate appropriate treatment for prevention of adverse cardiovascular outcomes, during and after liver transplantation, a high-risk surgical procedure. 
Funding information The authors state that this work has not received any funding. Open access funding provided by University of Innsbruck and Medical University of Innsbruck.

\section{Compliance with ethical standards}

Guarantor The scientific guarantor of this publication is Prof. Gudrun Maria Feuchtner.

Conflict of interest The authors of this manuscript declare no relationships with any companies whose products or services may be related to the subject matter of the article.

Statistics and biometry One of the authors has significant statistical expertise.

Informed consent Written informed consent was waived by the Institutional Review Board.

Ethical approval Institutional Review Board approval was not required because it is a retrospective data analysis.

\section{Methodology \\ - Retrospective \\ - Observational \\ - Performed at one institution}

Open Access This article is licensed under a Creative Commons Attribution 4.0 International License, which permits use, sharing, adaptation, distribution and reproduction in any medium or format, as long as you give appropriate credit to the original author(s) and the source, provide a link to the Creative Commons licence, and indicate if changes were made. The images or other third party material in this article are included in the article's Creative Commons licence, unless indicated otherwise in a credit line to the material. If material is not included in the article's Creative Commons licence and your intended use is not permitted by statutory regulation or exceeds the permitted use, you will need to obtain permission directly from the copyright holder. To view a copy of this licence, visit http://creativecommons.org/licenses/by/4.0/.

\section{References}

1. VanWagner LB, Lapin B, Levitsky J et al (2014) High early cardiovascular mortality after liver transplantation. Liver Transpl 20: 1306-1316

2. Kong YG, Kang JW, Kim YK et al (2015) Preoperative coronary calcium score is predictive of early postoperative cardiovascular complications in liver transplant recipients. Br J Anaesth 114: 437-443

3. Jodocy D, Abbrederis S, Graziadei IW et al (2012) Coronary computer tomographic angiography for preoperative risk stratification in patients undergoing liver transplantation. Eur J Radiol 81:2260 2264

4. Bektas A, Sen R, Ferrucci L (2016) Does a bit of alcohol turn off inflammation and improve health? Age Ageing 45:747-748

5. Sadhu JS, Novak E, Mukamal KJ et al (2018) Association of alcohol consumption after development of heart failure with survival among older adults in the cardiovascular health study. JAMA Netw Open 1:e186383

6. Wood AM, Kaptoge S, Butterworth AS et al (2018) Risk thresholds for alcohol consumption: combined analysis of individual- participant data for 599912 current drinkers in 83 prospective studies. Lancet 391:1513-1523

7. SCOT-HEART Investigators, Newby DE, Adamson PD et al (2018) Coronary CT angiography and 5-year risk of myocardial infarction. N Engl J Med 379:924-933

8. Thomsen C, Abdulla J (2016) Characteristics of high-risk coronary plaques identified by computed tomographic angiography and associated prognosis: a systematic review and meta-analysis. Eur Heart J Cardiovasc Imaging 17:120-129

9. Maurovich-Horvat P, Schlett CL, Alkadhi $\mathrm{H}$ et al (2012) The napkin-ring sign indicates advanced atherosclerotic lesions in coronary CT angiography. JACC Cardiovasc Imaging 5:1243-1252

10. Feuchtner G, Kerber J, Burghard P et al (2017) The high-risk criteria low-attenuation plaque $<60 \mathrm{HU}$ and the napkin-ring sign are the most powerful predictors of MACE: a long-term follow-up study. Eur Heart J Cardiovasc Imaging 18:772-779

11. Kim HJ, Lee HW (2013) Important predictor of mortality in patients with end-stage liver disease. Clin Mol Hepatol 19:105-115

12. American Psychiatric Association (2013) Diagnostic and statistical manual of mental disorders. https://doi.org/10.1176/appi.books. 9780890425596.dsm16

13. European Association for the Study of the Liver (2018) EASL clinical practice guidelines: management of alcohol-related liver disease. J Hepatol 69:154-181

14. Agatston AS, Janowitz WR, Hildner FJ, Zusmer NR, Viamonte M Jr, Detrano R (1990) Quantification of coronary artery calcium using ultrafast computed tomography. J Am Coll Cardiol 15:827832

15. Cury RC, Abbara S, Achenbach S et al (2016) CAD-RADS(TM) Coronary Artery Disease - Reporting and Data System. An expert consensus document of the Society of Cardiovascular Computed Tomography (SCCT), the American College of Radiology (ACR) and the North American Society for Cardiovascular Imaging (NASCI). Endorsed by the American College of Cardiology. J Cardiovasc Comput Tomogr 10:269-281

16. Austen WG, Edwards JE, Frye RL et al (1975) A reporting system on patients evaluated for coronary artery disease. Report of the Ad Hoc Committee for Grading of Coronary Artery Disease, Council on Cardiovascular Surgery, American Heart Association. Circulation 51:5-40

17. Feuchtner GM, Barbieri F, Langer C et al (2019) Non obstructive high-risk plaque but not calcified by coronary CTA, and the Gscore predict ischemia. J Cardiovasc Comput Tomogr. https://doi. org/10.1016/j.jcct.2019.01.010

18. Leber AW, Knez A, Becker A et al (2004) Accuracy of multidetector spiral computed tomography in identifying and differentiating the composition of coronary atherosclerotic plaques: a comparative study with intracoronary ultrasound. J Am Coll Cardiol 43: 1241-1247

19. Tomizawa N, Inoh S, Nojo T, Nakamura S (2016) Relationship of hepatic steatosis severity and coronary artery disease characteristics assessed by coronary CT angiography. Int J Cardiovasc Imaging 32(Suppl 1):73-82

20. Kazankov K, Munk K, Ovrehus KA et al (2017) High burden of coronary atherosclerosis in patients with cirrhosis. Eur J Clin Invest 47:565-573

21. McAvoy NC, Kochar N, McKillop G, Newby DE, Hayes PC (2008) Prevalence of coronary artery calcification in patients undergoing assessment for orthotopic liver transplantation. Liver Transpl 14:1725-1731

22. Mouton AJ, Ninh VK, El Hajj EC, El Hajj MC, Gilpin NW, Gardner JD (2016) Exposure to chronic alcohol accelerates development of wall stress and eccentric remodeling in rats with volume overload. J Mol Cell Cardiol 97:15-23 
23. Rodrigues P, Santos-Ribeiro S, Teodoro T et al (2018) Association between alcohol intake and cardiac remodeling. J Am Coll Cardiol 72:1452-1462

24. Larsson SC, Wallin A, Wolk A (2018) Alcohol consumption and risk of heart failure: meta-analysis of 13 prospective studies. Clin Nutr 37:1247-1251

25. Hell MM, Motwani M, Otaki Y et al (2017) Quantitative global plaque characteristics from coronary computed tomography angiography for the prediction of future cardiac mortality during longterm follow-up. Eur Heart J Cardiovasc Imaging 18:1331-1339

26. Rizvi A, Hartaigh BO, Danad I et al (2017) Diffuse coronary artery disease among other atherosclerotic plaque characteristics by coronary computed tomography angiography for predicting coronary vessel-specific ischemia by fractional flow reserve. Atherosclerosis 258:145-151

27. Ahmadi A, Leipsic J, Ovrehus KA et al (2018) Lesion-specific and vessel-related determinants of fractional flow reserve beyond coronary artery stenosis. JACC Cardiovasc Imaging 11:521-530

28. van Rosendael AR, Narula J, Lin FY et al (2020) Association of high-density calcified $1 \mathrm{~K}$ Plaque with risk of acute coronary syndrome. JAMA Cardiol. https://doi.org/10.1001/jamacardio. 2019.5315

29. Xie JX, Cury RC, Leipsic J et al (2018) The Coronary Artery Disease-Reporting and Data System (CAD-RADS): prognostic and clinical implications associated with standardized coronary computed tomography angiography reporting. JACC Cardiovasc Imaging 11:78-89

30. Han D, Hartaigh BO, Gransar H et al (2018) Incremental prognostic value of coronary computed tomography angiography over coronary calcium scoring for major adverse cardiac events in elderly asymptomatic individuals. Eur Heart J Cardiovasc Imaging 19: 675-683

31. Ripoll C, Yotti R, Bermejo J, Banares R (2011) The heart in liver transplantation. J Hepatol 54:810-822

32. Williams MC, Moss AJ, Dweck M et al (2019) Coronary artery plaque characteristics associated with adverse outcomes in the SCOT-HEART study. J Am Coll Cardiol 73:291-301

Publisher's note Springer Nature remains neutral with regard to jurisdictional claims in published maps and institutional affiliations. 